\title{
Highlights of the AAPS Workshop on Dissolution Testing for the 21st Century
}

\author{
Jan Parker ${ }^{1,3}$ and Vivian Gray ${ }^{2}$
}

e-mail:jparker@lancasterlabs.com

From May 1-3, 2006, the AAPS In Vitro Release and Dissolution Testing Focus Group sponsored an interesting and exciting workshop on Dissolution Testing for the 21st Century, Linking Critical Quality Attributes and Critical Process Parameters to Clinically Relevant Dissolution Method/ Specification. Approximately 232 people from diverse disciplines attended the meeting, held in Arlington, Virginia. The attendees included engineers, pharmacists, chemists, regulators, and researchers. There were also many vendors present to exhibit their equipment and answer questions.

The presentations provided a wide variety of opinions on dissolution testing, and there was a panel discussion after each session to provide participant insights and questions. Tahseen Mirza, Ph.D., Chair of the In Vitro Release and Dissolution Testing Focus Group, opened the meeting and outlined the first day's session that was on dissolution as it relates to the quality by design (QBD) /process analytical technology (PAT) initiatives by FDA. The presentations on day two focused on new dosage forms, the method development challenges associated with new products, and the expectations for clinically relevant specifications. The last day of the workshop was highlighted by presentations that discussed the merits of calibrator tablets and mechanical calibration, including a USP initiative on new acceptance criteria for both products and calibration.

\section{Session I and II: PAT and its Relevance to Dissolution}

The first speaker was Mohab Nasr, Ph. D., FDA, who set the tone for the conference. In his talk, "Quality by design and its relevance to dissolution," he began by listing the possible role of dissolution testing. This role is to guide drug development in selecting formulations, bridging clinical studies, surving as a surrogate for in vivo bioequivalence, and providing quality control for batch-to-batch consistency, stability, and support biowaivers for the Biopharmaceutics Classification System (BCS) Class I. He examined the current system for setting specifications where (1) there is an empirical approach used to fit the available data, (2) specifications are set through negotiation, (3) specifications are estab- lished late in the approval process, and (4) the linkage to safety and efficacy is not always assured. He went on to explain that specifications may not be reflective of "true" product quality; out-of-specification results may lead to production delays, inefficiency, and drug shortages, and setting specifications is a regulatory hurdle that needs improvement. The limitations of the current system were highlighted with an emphasis on lack of adequate product and process understanding. The dissolution test may not be a sensitive indicator of product performance for dosage forms such as highly soluble and highly permeable drugs or potent or narrow therapeutic index drugs with low solubility. He posed the question, "Could disintegration or some other quality attributes substitute for dissolution?"The desired state is to have a knowledge-rich submission, one that understands and controls variation with specifications based on product performance requirements. In summary, his message was that dissolution may play a more important role in QbD with drug development by providing accurate clinical linkage and less as a quality control test. He expressed the FDA's willingness to work with scientific organizations such as the AAPS focus group, PQRI, universities, and the USP.

To give the industry perspective on the future of dissolution, Yatindra Joshi, Ph.D, Novartis Pharmaceuticals, presented his views in the talk, "Pharmaceutical development in the 21 st century." He looked at the future of technical development where development time lines will become even more aggressive with more frequent use of modeling, in vitro methods for prediction of in vivo behavior, PAT, robotics and automation, and elimination of scale-up risk. He pointed out some dissolution issues that need further exploration such as hydrodynamic effects, low volume dissolution testing, increased speed as an in-process test, and overencapsulation.

A case study using PAT and dissolution was presented by Kimberly Gallagher and Robert Green, Merck Research Laboratories, "Process analytical techniques and dissolution: a Merck case study using surrogates for particle-size mediated dissolution mechanisms." The case study presented 
used a BCS Class IV compound where the feasibility of using particle size analysis and NIR as a surrogate for dissolution testing was evaluated. The use of the entire particle size distribution (PSD) of the overencapsulated formulation contents using laser diffraction of the sieved contents proved to have observations consistent with the dissolution data. The NIR studies were quite useful, satisfying all release requirements, composite assay, dosage form uniformity, identity, and dissolution. In addition, the technique gave information on lubricant level and moisture content. The conclusion was that for the BCS Class IV, both PSD and NIR were suitable surrogates for dissolution analysis with NIR providing an attractive real-time release option.

The subject of NIR was further explored by Fiona Clarke, Ph.D., Pfizer. Her talk, "Building understanding of dissolution behavior using near-infrared chemical images: A prediction of performance,"illustrated how chemical images can be used to provide a mechanistic understanding of how formulation and process factors influence product performance. The visualization offered by NIR is a good means of problem detection. In the search for a root cause, the dissolution test can tell you there is a change but not what has changed. The images can provide information on component distribution, size, or matrix change. The NIR chemical images have been used to troubleshoot, build understanding of the dissolution performance, understand processing effects on formulation changes, provide a design space for dissolution based on process DoE, and show a direct correlation to dissolution performance. Some case studies were presented. In the future, this technology has the potential to be used to predict dissolution performance, and at the blend stage, the analyst can understand if the same matrix has been produced. A scientific understanding of dissolution properties can be obtained from NIR chemical images.

After a panel discussion, the second session devoted to PAT and its relevance to dissolution was begun with a talk by Qingxi Wang, Ph.D., Merck \& Company, "A quality-bydesign approach to dissolution based on the biophamaceutical classification system." Dr. Qingxi presented ten case studies that illustrated Merck's experiences with many different dosage forms and the eventual relevance of the dissolution test. His conclusions were that enhanced science and risk-based regulatory quality assessment is possible for drug release testing, possibly leading to removal of non-value-added specifications and reduction of the volume of data generated and submitted. In the case of BCS Class I/III immediate release (IR) products, there may be the ability to eliminate dissolution testing and replace it with disintegration testing. For BCS Class II/IV IR products, there may be key quality attributes that could replace the dissolution test, although some dissolution testing still adds value. In the case of extended-release (ER) products, the dissolution test still adds value but may be challenged in the future.

The next speaker, John Kirsch, Ph. D, Mylan Pharmaceuticals, also spoke on NIR in his talk, "The utility of nearinfrared spectroscopy in the prediction of dissolution behavior." He discussed the caveats that go along with using NIR. Numerous literature examples have demonstrated NIR's ability to predict dissolution and other tablet properties, but the physiological relevance of these properties will, for the most part, remain unknown. Does this increased sensitivity make NIR better, worse, or merely complementary to dissolution as a tool for ensuring the quality of pharmaceutical products? He presented several cases of NIR evaluation of dissolution and related tablet properties. Near infrared may not be best applied by studying spectral correlations with a single dissolution parameter. Instead, a composite assessment of the conformance of the dosage form to individual Critical Quality Attributes (CQA) may be preferred. Several case studies dealt with predicting tablet hardness, assay, and coating levels from NIR.

The last speaker before the afternoon panel discussion was Gary Ritchie, Ph. D., USP, speaking on "PAT and its relevance to dissolution - a USP perspective."He began his presentation with a discussion of bioequivalence (BE) concepts and how equivalence is of utmost importance to the patient. The dissolution test is related to $B E$ and bioavailability (BA) only when closely allied with a sound regulatory determination. Without this link, the USP performance test (the dissolution test) should be regarded solely as a quality control test for batch release. He made several points regarding PAT:PAT is not trivial as many but not all of the techniques rely on skills in chemometric design and modeling techniques, a deep understanding of the physical and chemical properties of the process composition materials are needed for meaningful correlations to be made, and finally these correlations from a model or other responses to a critical process parameter must be validated. This validation may not be possible in all cases. Multivariate models are intended as surrogates for process measurement and control, not release. The final product should require a separate and distinct model. He emphasized that analytical approaches intended for measurement and control for manufacturing processes are not intended to correlate to in vivo drug kinetics. He discussed the suitability of NIR chemical imaging $(\mathrm{Cl})$ to correlate with dissolution, pointing out that in diffuse reflectance mode, $\mathrm{NIR}-\mathrm{Cl}$ is used for blend uniformity assessment and in transmission mode, $\mathrm{NIR}-\mathrm{Cl}$ may have extended applicability. To address these topics, the USP has established an advisory panel for the USP General Chapter <1119> on Near-Infrared Spectroscopy. There is also the expectation that there will be new USP informational general chapters on chemical imaging/ multivariate analysis and chemometrics by the end of 2006 . He concluded with the statement that since PAT is an alternative procedure, the USP monograph including the dissolution test remains the referee method.

\section{Session III: Dissolution of Novel Dosage Forms}

The session began with the FDA perspective on the dissolution of novel dosage forms, presented by Angelica Dorantes, Ph.D., Office of Clinical Pharmacology, Center for Drug Evaluation and Research,FDA. She began by 
reviewing the types of novel dosage forms and stating that the same general principles that apply to conventional oral dosage forms apply to novel ones. Due to the manner in which these dosage forms release drug in the body, the term "dissolution" may be replaced with "drug release" or "drug elution." However, the roles that the test plays are the same as with conventional oral dosage forms. Like dissolution, drug release testing aids in formulation development, ensures release of a quality product to market, and can serve as an in vivo surrogate test. The latter function can be especially important with novel dosage forms since bioequivalence testing may be difficult to perform. Because the goals are the same regardless of dosage form, the method development considerations for drug release testing are also the same. The release profile should reach $80 \%$ of label claim or at least reach a plateau. A profile that mimics the in vivo profile is especially desirable. It is important that the method be QC-friendly. The specifications should not be set to accommodate variability of the product but to ensure bioequivalence of all lots meeting the elution specifications. One difference between traditional dissolution testing and the drug release testing of novel dosage forms is that Apparatus 1 and Apparatus 2 are often not the best apparatus to use in testing these types of products. The other compendial dissolution apparatus are often better suited for the dosage form under consideration and should be utilized if appropriate. Modifications to conventional apparatus, such as minivessels and paddles for Apparatus 2, may also be acceptable. Non-conventional media, such as hydroalcoholic media, may be required. Although the FDA accepts that these dosage forms present special challenges and may require non-traditional approaches, it is crucial to have interaction with FDA throughout the method development and validation process. FDA expects an elution method regardless of dosage form, and communication between the drug developer and the agency is the key to meeting this requirement.

An example of a novel dosage form that requires a modified apparatus for testing was the subject of the next speaker's talk. Johannes Krämer, Ph.D., PHAST Gmbh described the European apparatus that have been designed for drug release testing of medicated chewing gums. Medicated gums offer some unique advantages as dosage forms, such as the ability to discontinue drug absorption instantly simply by having the patient remove the gum. The patient also has some control over the delivery time based on the rate of chewing. None of the USP compendial apparatus can mimic the in vivo chewing mechanism, and release is dependent on very strong mechanical forces. The European Pharmacopoeia has a compendial apparatus designed especially for medicated gums and Dr. Krämer presented data collected using the apparatus. He also showed slides of a second apparatus that is commercially available. The European Pharmacopoeia does not yet give any performance qualification specifications for the chewing gum tester, and discussions are continuing on the subject.

The drug-eluting stent (DES) is another novel dosage form that challenges the dissolution scientist. Stephen P.Mayock,
Cardinal Health examined some of the challenges associated with drug release testing of DES. He compared the advantages and disadvantages of Apparatus 7, Apparatus 4, Apparatus 2 equipped with mini-vessels, and the noncompendial incubated agitation method. Because the drugs are often sparingly soluble in water, determining the drug solubility at various $\mathrm{pH}$ s and in aqueous media containing surfactants is critical to developing the drug release method. Hydroalcoholic media may need to be investigated. Whatever medium is chosen, it is important to maintain sink conditions throughout testing. Although drug release testing is generally performed at $37^{\circ} \mathrm{C}$, it can be advantageous to increase the temperature in order to speed up drug release; however, increasing the temperature will also increase evaporation and possibly increase extraction of interfering compounds from the plastic parts that contact the heated medium. Extraction from plastic is also a concern when using hydroalcoholic media. While IVIVR is always one of the goals of drug release testing, it can be very difficult to establish IVIVR with DES because the drug is not released systemically in vivo. Retrieving implanted stents and analyzing them for remaining drug indicates the amount of drug that has been released, but this type of study is generally limited to animal subjects.

Michael K.Taylor, Ph. D., GlaxoSmithKline spoke on using dissolution as a screening tool during the development of engineered, sustained-release inhalation particles. The dissolution test was used in comparing different formulations during product development. The product was designed to be sustained release and to target a specific region of the lung. Sustained-release properties could result either from the product matrix or from coating the particles. A release method developed using USP Apparatus 4 was used to guide the particle-engineering process. While the dissolution profiles did not mimic in vivo exactly, a relationship that could serve as the initial step to IVIVR was established.

Apparatus 4 was the focus of the presentation given by David Baum, Ph.D., Acumen Pharma Consulting. He examined the advantages of using Apparatus 4 in drug release testing for implants as well as DES. One of the many inherent challenges of drug devices is that the drug load on the product can be very small, requiring low volumes of medium in order to maintain sensitivity during the analytical finish. Although Apparatus 4 was originally designed as an open system with the dosage unit having continuous exposure to fresh medium, it can be converted into a closed loop by using individual media reservoirs for each of the channels and having the waste tubing returning to these same reservoirs. This allows the use of low volumes of dissolution medium and minimizes loss due to evaporation since the system is a closed loop. This is especially important for these devices because extended run times can be required to achieve a plateau in the elution profile. Another advantage of Apparatus 4 is the availability of a variety of sample cells that have been designed for specific novel dosage forms. Not all of the cells are compendial, but the product 
manufacturer can often justify the use of non-compendial equipment by communicating with FDA early in the product development.

\section{Session IV: Challenges in Dissolution Testing}

The FDA perspective on the challenges in dissolution testing for the 21 st century was given by Lawrence $\mathbf{X}$. Yu, Ph.D., Office of Generic Drugs, FDA. He began by addressing some of the issues with the current dissolution test and bioequivalence. The test can sometimes be nondiscriminating by failing to detect a difference in products that are not bioequivalent and sometimes over-discriminating by failing products that are in fact bioequivalent. One issue is that single-point dissolution tests often do not adequately reflect in vivo behavior. Another is that IVIVR cannot always be demonstrated, and when it is, the correlation is only for the sponsor's product. These issues are related to the way the drug is absorbed by the body, depending on whether absorption is dissolution-limited, solubilty-limited, or permeability-limited. Part of the problem may be expecting the dissolution test to detect batch-to-batch inconsistencies in a QC setting and yet not be so sensitive that it fails bioequivalent batches for biowavers. Dr. Yu proposed designing a single biorelevant medium that would be used for all products, at least for purposes of bioequivalence. This would permit direct comparison among similar products from different manufacturers and streamline dissolution testing in the analytical laboratory. Further study is needed to determine whether the biorelevant medium would be acceptable for QC testing. The purpose of the release dissolution test is to detect inconsistencies in the product, some of which might not be biorelevant but might still be unacceptable in the finished product.

Representing the generic industry's views on the same topic was John Kovaleski, Ph.D., Teva Pharmaceuticals USA. Since generic products are bioequivalent to the brand-name drug by definition, the generic drug must meet the same dissolution requirements as the brandname product. This means that the generic competitor is not free to develop a dissolution method based on its own generic formulation. If the product is USP, the generic company is effectively locked into the USP requirements; if it is not USP, the generic company must turn to the method supplied by the Office of Generic Drugs (OGD.) Many times the " $\mathrm{Q}$ " value is not supplied, and there is uncertainty on setting the specifications. Since the goal of the QbD is to build quality into the product beginning with the product development stage, the generic industry would like to design dissolution tests around its own products rather than have the tests be handed down from another manufacturer. The critical attributes for each manufacturer may be different due to excipients or manufacturing processes, and each manufacturer should be able to design dissolution tests that are the best measure of these critical attributes. OGD has recently implemented a Question Based Review (QBR) system that will allow generic companies to justify the tests and acceptance criteria that they develop specifically for their products. This approach may allow the generic industry to develop more meaningful dissolution methods.

Comparators present some unique dissolution challenges for method development and validation. This topic was addressed by Diane Worrell, Bristol-Myers Squibb with input from Jamie Bell, Aptuit and Ruben Lozano, BristolMyers Squibb. Comparator dissolution methods may be available through compendial sources, or it may be possible to access the method filed for the brand name product using the Freedom of Information Act. If neither of these options is available, a method will have to be developed. The FDA Guidance for Industry, Dissolution Testing of Immediate Release Solid Dosage Forms, can be used as a guideline in developing a method. The validation of a comparator method consists of the same components as a typical dissolution validation, but it also requires profile comparison with the innovator product. A general dissolution method validation strategy for performing validation in the dissolution vessel was presented in which specificity, linearity, accuracy, and repeatability are performed simultaneously in the dissolution bath per the method. Pre-validation experiments during method development were also recommended. Some of the challenges presented by overencapsulated comparators include pellicle formation of the capsules, the failure of the $F 2$ comparison due to capsule lag at early time points, and the lack of a commercially-available reference standard. Pellicle formation can be overcome with the addition of enzymes to the medium as described in USP $<711>$. Reference standard could be isolated from the innovator product (in high-strength formulations) or synthesized; often a composite of the innovator product is used as a"pseudostandard." Some of the unresolved issues with comparator testing are the specification-setting process and the degree to which a compendial method can be modified in order to accommodate overencapsulated product.

Method development and validation requirements vary with the phase of product development. Saji Thomas, M. Sc., Barr Laboratories described a strategy for phaseappropriate development and validation. First, he described how the roles played by the in vitro dissolution test vary with the stages in a product life cycle. For early phase development, the only robustness needed is solution stability; but in late phase development, parameters for method robustness need to be established. Then he demonstrated how robustness could be evaluated by using Design of Experiments (DoE) principles to define a design space. The advantage of defining a design space is that working within a design space is not considered a change. For dissolution robustness, independent variables such as bath temperature, paddle speed, $\mathrm{pH}$ and salt concentration of the medium, and analytical wavelength can be varied in a multifactor-designed matrix experiment. The dependent variable is the dissolution result. Statistical analysis of the results identifies which parameters are critical to control and demonstrate how rugged or non-rugged the method is. 
Alex Shlyankevich, Ph. D., Bioavail Technologies wrapped up the afternoon session with an enlightening talk,"Misinterpretation of Dissolution Data and Its Impact on Product Development." He shared three anecdotal situations in which wrong conclusions were reached by taking logical steps based on dissolution results. In all three cases, the right conclusions were eventually reached by taking time to observe what was happening to the product in the vessel during dissolution. His experiences emphasize the importance of making careful and accurate observations during dissolution testing.

\section{Session V: Dissolution - Hot Topics}

The first of the dissolution "hot topics" was the setting of dissolution specifications, both for products and for the performance verification tablets (formerly referred to as "calibrator tablets"). Walter W.Hauck, Ph.D, Thomas Jefferson University, USP Statistical Consultant addressed the topic of setting dissolution specifications based on statistical approaches. He outlined a method for setting specifications based on the distribution of dissolution results in clinically relevant batches. Then he described how USP arrives at the current specifications for the performance verification tablets. The current requirements set acceptance criteria per tablet, but all six tablets tested must pass. This increases the rate of false positives above that expected based on a single tablet. A proposed specification for product verification would base the results on the mean and standard deviation of the tablets tested. The assigned value and standard deviation used as the window for passing results would still be determined by the collaborative study used currently. USP is also looking at ISO guides and considering how closely they could be applied in setting performance verification tablet specifications.

The role of hydrodynamics in dissolution testing was discussed by Tahseen Mirza, Ph.D., Novartis. He shared the results of a series of experiments that examined the effect of vessel type and paddle speed on cone formation. The presence of a dead zone under the paddle in a typical dissolution vessel was demonstrated by deliberately tilting the vessels so that the cone would be subjected to increased agitation and disperse. Once it was established that the dead zone existed, minimizing its impact by either using Peak ${ }^{\mathrm{TM}}$ vessels or increasing the paddle speed was investigated. Both were found to be about equally effective. Then the consequences of increasing paddle speed on discrimination were examined. By running a series of paddle-speed experiments on the same product in USP and Peak ${ }^{T M}$ vessels, the proper balance between discrimination and paddle speed was achieved. Under the particular conditions used for the product studied, the optimum paddle speed in USP vessels was 60 rpm, while in Peak ${ }^{\mathrm{TM}}$ vessels, 40 rpm was ideal.

The FDA representative for the topic of mechanical calibration was Lucinda Busche, Ph.D., Acting Director of the Office of Testing and Reasearch, In her talk, "Dissolution Apparatus Qualification," she stated that the goal of her presentation was to point out the sources of variability and describe how to set up and operate apparatus to minimize uncertainty. Variability can be caused by the instrument suitability including such aspects as the operator, apparatus variability, and calibrator tablet variability. Calibrator tablet variability, according to Dr. Busche, can be assigned to manufacturing and/or stability of the tablet, instrument setup, and degassing. There are drug-product-specific sources of variability such as the media (including degassing), product manufacturing, dissolution equipment parameters, sinkers, and the determinative step. She gave alternative approaches to dissolution calibration and validation. The analyst needs to perform stringent mechanical calibration to replace the need for calibrator tablets. All sources of variability need to be identified and controlled. The interaction between the instrument and product needs to be understood. If necessary, establish an internal reference (biobatch or clinical batch) for system suitability and stability; the suitability of this reference product would be confirmed using Gage R \& R. This internal reference developed from a clinical or biobatch may be more applicable to the product than the dissolution calibrators. She presented data indicating that vessel asymmetry and centering can influence the dissolution results. Two-point centering was used to ensure verticality. A table of mechanical calibration tolerances was shown. Vibration, hydrodynamics, and new approaches to assess drug release will be the topics for future research.

Bryan Crist from Varian gave a talk on "Evaluation of induced variance of physical parameters on the calibrated USP dissolution apparatus 1 and 2." This presentation focused on the variance of several physical parameters and configurations to demonstrate their effect on Apparatus 1 and 2. The USP Prednisone Disintegrating Calibrator Tablet was used. Several parameters in particular were studied: vessel temperature, vibration, vessels, and basket mesh size and attachment configuration. The tablets were not sensitive to temperatures ranging from $36.5^{\circ} \mathrm{C}$ to $37.5^{\circ} \mathrm{C}$. A displacement of 0.02 mils and a frequency of $130 \mathrm{~Hz}$ showed a noticeable effect on the dissolution results. The use of different basket attachments should be evaluated versus the official clip-type attachment. He pointed out that deformities and irregularities in the vessel may not be detected with the fingertips and vision alone. He stated that the vessel hemisphere should be defined with a specific tolerance.

The meeting was concluded with a talk by Vivian Gray, $\mathbf{V}$. A.Gray Consulting,"Current challenges to dissolution testing, including calibrators, rebuttal and common ground." The discussion points were the choice of clinically relevant specifications, vessel hydrodynamics and flow, and mechanical and chemical calibration. She shared the view that PAT should be beneficial to the industry as it will bring more in-depth knowledge of the drug, excipients, and manufacturing process. Method development should be more thorough, and methods should be as clinically relevant as possible including an increased emphasis on discriminating methods and/or in vivo and in vitro correlations and relationships. She mentioned that USP has a new 
official General Chapter <1092> called The Dissolution Procedure:Development and Validation. This chapter gives guidance on developing discriminating methods. The hydrodynamics and fluid flow should be observed during testing, and if undisturbed homogenous-type mixing is not present, the result may be affected adversely. Calibration using the USP Calibrator tablets was strongly encouraged as the calibrator tablets so far are the only way to determine if the equipment is prone to significant vibration and vessels with flaws. The performance test with the calibrator tablets can show synergistic errors and also covers 30 minutes rather than a snap-shot-in-time mechanical calibration. She stressed that doing away with the use of calibrator tablets weakens the standard for apparatus suitability, paving the way for use of dissolution equipment that is below par.

The session ended with a lively panel discussion. Saji

Thomas stated that the proceedings with summaries of the panel discussion would be published in a manuscript, and a book of the presentations is under consideration. He thanked the participants for making the meeting very worthwhile through their comments and questions.

For a limited time, the presentation slides are on the AAPS website at this link http://www.aapspharmaceutica.com/ meetings/meeting.asp?id=63. All those interested in joining the AAPS In Vitro Release and Dissolution Testing Focus group may do so by going to the AAPS website. 